\title{
Avances en el conocimiento de la diversidad de la familia Brassicaceae en Ancash, Perú
}

\section{Advances in the diversity knowledge of Brassicaceae family in Ancash, Peru}

\author{
Christhian Monsalve ${ }^{1}$ y Asunción Cano ${ }^{1,2}$
}

Presentado: $18 / 11 / 2004$

Aceptado: $\quad 12 / 05 / 2005$

\section{Resumen}

Se confirma la presencia de 40 especies de plantas de la familia Brassicaceae, agrupadas en 17 géneros y cinco tribus en el departamento de Ancash, Perú. Del total de especies, tres son consideradas endémicas de Ancash y dos son endémicas nacionales. Se presenta claves taxonómicas para la identificación de generos y especies así como datos de distribución para cada especie.

Palabras clave: Perú, Ancash, Brassicaceae, composición florística, endemismo.

\section{Abstract}

The presence of 40 species of plants of the Brassicaceae family grouped in 17 genera and 5 tribes is confirmed within the area of study. From the total of the species, three are considered endemic of Ancash and two are nationally endemics. Dichotomous keys for the genera and species are presented as well as distribution information for each of the species.

Key words: Peru, Ancash, Brassicaceae, floristic composition, endemism.

\section{Introducción}

La flora de Ancash no ha sido detallada en su totalidad hasta la fecha. La información existente acerca de la familia Brassicaceae corresponde principalmente a los trabajos realizados por Cerrate (1979) quien reportó la flora de Chiquián al sur de Ancash, Smith (1988) quien realizó un inventario florístico en el Parque Nacional Huascarán y Albán (1998) que realizó un estudio etnobotánico en la comunidad andina de Pamparomás ubicada en la provincia de Huaylas. Trabajos más generales, y de los cuales se puede obtener información acerca de las especies de Brassicaceae presentes en Ancash son los de Macbride (1938) en Flora of Peru, y Brako \& Zarucchi (1993) en el Catálogo de las Angiospermas y Gimnospermas del Perú.

${ }^{1}$ Museo de Historia Natural, Universidad Nacional Mayor de San Marcos. Avenida Arenales 1256, Lima 11, Apartado 14-0434, Perú.

Christhian Monsalve: christhianml@hotmail.com

${ }^{2}$ Facultad de Ciencias Biológicas, Universidad Nacional Mayor de San Marcos.
En trabajos anteriores (Monsalve y Cano, 2003; Cano et al., 2000) se destacó la importancia de la familia Brassicaceae en los ecosistemas altoandinos debido a que es una de las familias de plantas vasculares con mayor número de especies, entre las cuales se encuentran algunas endémicas del departamento de Ancash conocidas sólo de sus respectivas localidades tipo. Tanto la importante diversidad de especies como la restringida distribución de algunas de ellas, hacen que sea necesario continuar realizando inventarios florísticos y estudios taxonómicos de esta familia para un mejor conocimiento de la flora altoandina del Perú.

En el Catálogo de las Angiospermas y Gimnospermas del Perú (Brako \& Zarucchi, 1993) la familia Brassicaceae está representada en Ancash por 30 especies y 17 géneros. Monsalve y Cano (2003) realizaron un estudio taxonómico de la familia Brassicaceae en la provincia de Huaylas de este departamento, encontrando 25 especies y 12 géneros e incrementando así el registro de esta familia para todo el departamento en 38 especies y 17 géneros. 
Según Brako y Zarucchi (1993), nueve especies de Brassicaceae son endémicas de Ancash, de un total de 28 en todo el Perú. Sin embargo, Monsalve y Cano (2003) no registraron ninguna en Huaylas. Entre algunas de estas especies endémicas destaca la presencia de dos géneros monotípicos Catadysia y Dactylocardamum.

El presente trabajo contribuye al conocimiento de la familia Brassicaceae proporcionando una relación actualizada de las especies presentes en el departamento, así como claves de identificación taxonómica y datos de distribución que sirvan de base para futuros estudios.

\section{Área de estudio}

El departamento de Ancash se encuentra ubicado entre los $8^{\circ} 00^{\prime} 01^{\prime \prime}$ y $10^{\circ} 45^{\prime} 001^{\prime \prime}$ de latitud sur y $76^{\circ} 45^{\prime} 00^{\prime \prime}$ y $78^{\circ} 39^{\prime} 00^{\prime}$ ' de longitud oeste; su capital, Huaráz, se encuentra a $400 \mathrm{~km}$ al norte de Lima. Está dividido políticamente en 20 provincias, siendo el departamento con mayor número de provincias en Perú. Estas son: Aija, Antonio Raimondi, Asunción, Bolognesi, Carhuaz, Carlos F. Fitzcarrald, Casma, Corongo, Huaraz, Huari, Huarmey, Huaylas, Mariscal Luzuriaga, Ocros, Pallasca, Pomabamba, Recuay, Santa, Sihuas y Yungay (Fig. 1).

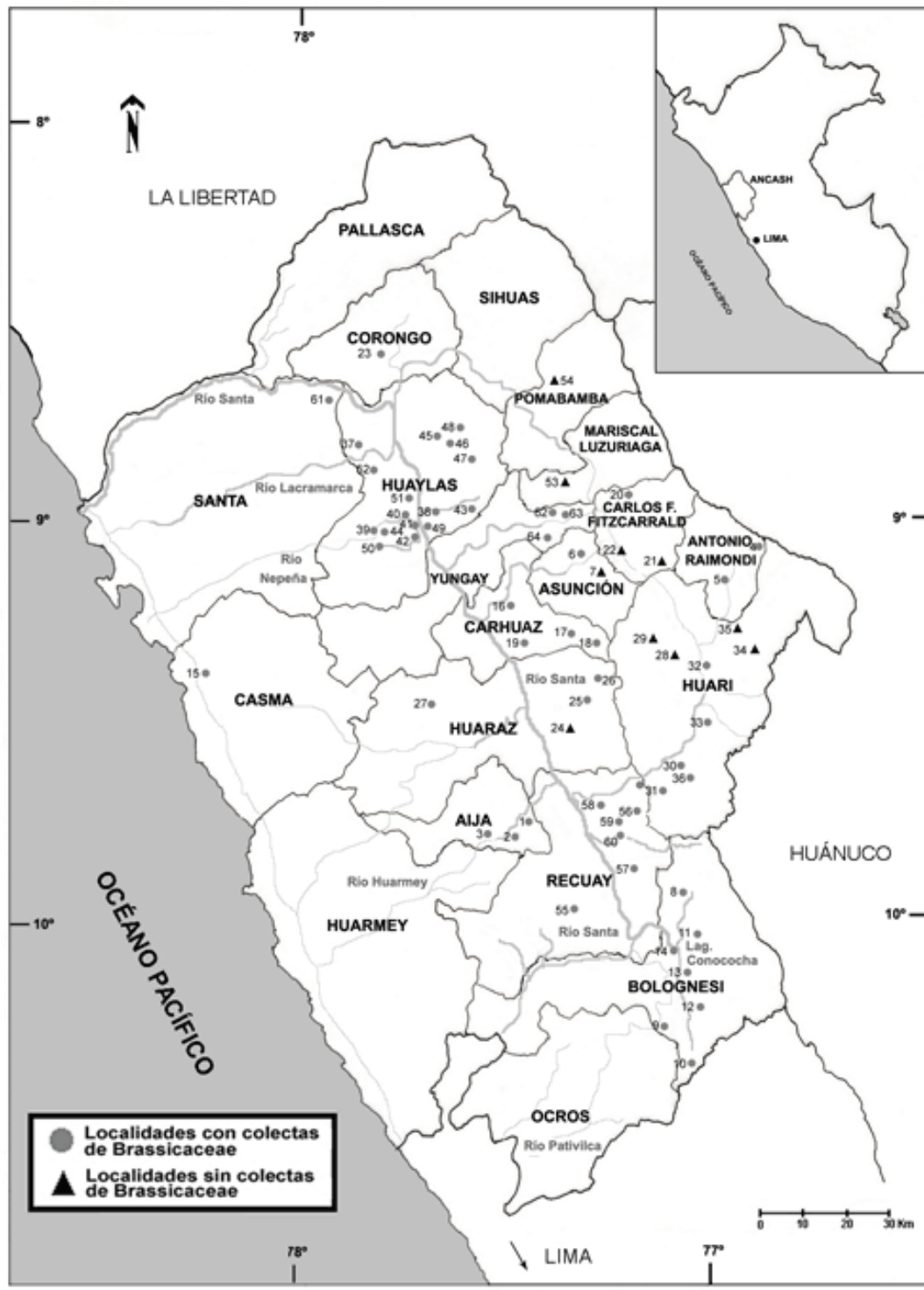

Figura 1. Mapa polítiAncash mostrando localidades de colecta. co del departamento de 
Presenta una diversidad de climas y hábitats que van desde el desierto costero hasta las heladas punas de las Cordilleras Blanca y Negra, los cuales están comprendidos en un amplio rango altitudinal desde cero hasta más de $5000 \mathrm{~m}$, con una precipitación promedio anual de $900 \mathrm{~mm}$. Tal diversidad de condiciones ambientales genera igualmente una importante diversidad de vegetación, la mayoría de la cual está representada y conservada en el Parque Nacional Huascarán que se extiende en casi la totalidad de la Cordillera Blanca (Peñaherrera, 1969).

\section{Materiales y Métodos}

El procesamiento y herborización de las plantas recolectadas se realizó siguiendo las técnicas estándares recomendadas (Cerrate, 1969; Lot y Chiang, 1986 y Bridson \& Forman, 1992).

El material analizado corresponde a recolectas realizadas por los autores y colaboradores entre los años 1999 y 2004 en un total de 64 localidades comprendidas en 16 provincias del departamento de Ancash, algunas de las cuales fueron visitadas durante las respectivas expediciones de recolecta, pero sin encontrar especie alguna (Anexo 1). También se incluyen los ejemplares recolectados por otros botánicos como D. N. Smith en el Parque Nacional Huascarán, J. Albán en Pamparomás, Huaylas y otros adicionales que forman parte de las colecciones del Herbarium Truxillense (HUT) de la Universidad Nacional de La Libertad-Trujillo y en el Herbario San Marcos (USM) de la Universidad Nacional Mayor de San Marcos.

\section{Resultados}

\section{Diversidad}

En el departamento de Ancash se registraron un total de 40 especies agrupadas en 17 géneros y cinco tribus (Anexo 2). El género con mayor número de especies es el género Draba con un total de nueve especies; mientras que los géneros Aschersoniodoxa, Capsella, Cardamine, Catadysia, Cremolobus, Englerocharis, Halimolobus, Mancoa y Nasturtium presentan solamente una especie cada uno. Así mismo, la tribu más diversa es la tribu Arabidae con 15 especies y seis géneros; y la tribu más pobremente representada es la tribu Brassiceae con seis especies y tres géneros.

Del total de especies que se registran para Ancash, cinco son endémicas. Estas especies son Catadysia rosulans O. E. Schulz, Draba ochropetala O. E. Schulz y Weberbauera smithii Al-Shehbaz, que se conocen exclusivamente de Ancash, mientras que Lepidium kalenbornii Hitchc. y Weberbauera perforata Al-Shehbaz se encuentran además en otros departamentos. Las especies $D$. ochropetala O. E. Schulz y $W$. perforata Al-Shehbaz han sido recolectadas nuevamente luego de la colección tipo.

Cinco especies tienen valor económico, la «maca» Lepidium meyenii Walp., el «berro» Nasturtium officinale R. Br., la «mostaza» Brassica nigra (L.) Koch, el «nabo» Brassica rapa subsp. campestris (L.) Clapham y el «rábano» Raphanus raphanistrum L. La «maca» L. meyenii Walp. es una especie inicialmente silvestre, pero que ha sido domesticada y adaptada al cultivo; mientras que las cuatro restantes son introducidas y tienen comportamiento de malezas, encontrándoseles generalmente como «invasoras» en campos de cultivo (Sagástegui, 1993).

\section{Tratamiento taxonómico}

Se sigue el sistema de clasificación de Cronquist (1981) según el cual, la posición taxonómica de la familia Brassicaceae es la siguiente:

\section{Clase Magnoliópsida \\ Subclase DilleniIdae \\ Orden Capparales \\ FAMILIA BRASSICACEAE (= CRUCIFERAE)}

Los principales caracteres morfológicos con valor taxonómico que sirven para identificar géneros y especies se dan en los frutos que pueden ser llamados silícuas o silículas dependiendo de su forma y dimensiones; las flores que pueden tener pétalos blancos, ama- 
rillos, rosados o lilas, o simplemente carecer de ellos; los tricomas que son muy variados, simples y alargados, bifurcados o estrellados; y el hábito, sufrutescente, herbáceo, cespitoso y pulviniforme.

\section{Clave para determinar los géneros de la familia Brassicaceae del departamento de Ancash}

1a. Fruto silícula, aplanada lateralmente.

1b. Fruto silícua, aplanada dorsi-ventralmente.

2a. Silícula con más de dos semillas por valva.

$$
\text { Capsella }
$$

2b. Silícula con una sola semilla por valva.

3a. Valvas orbiculares o suborbiculares frecuentemente pubescentes.

Cremolobus

3b. Valvas semi-circulares generalmente glabras.

\section{Lepidium}

4a. Plantas glabras o glabrescentes con tricomas alargados simples; silícuas divididas en porción valvar y estilar.

4b. Plantas pubescentes con tricomas bifurcados y estrellados; silícuas no divididas.

5a. Flores con pétalos amarillos; silícuas con porción valvar dehiscente.

Brassica

5b. Flores con pétalos blancos o púrpuras; silícuas con porción valvar indehiscente.

Raphanus

6a. Silícuas pequeñas, casi tan largas como anchas.

\section{Draba}

6b. Silícuas grandes, notablemente más largas que anchas.

7a. Hojas siempre bipinnadas.

7b. Hojas enteras de bordes variados o en ocasiones divididas pero nunca bipinnadas.

8a. Plantas muy ramificadas, generalmente erguidas; semillas humedecidas frecuentemente mucilaginosas.

\section{Descurainia}

8b. Plantas pequeñas con tallos postrados; semillas humedecidas nunca mucilaginosas. Mancoa

9a. Plantas herbáceas con hojas divididas y raíces adventicias en los nudos.

9b. Plantas herbáceas o sufrutescentes con hojas enteras y sin raíces adventicias en los nudos.(11)

10a. Silícuas desprovistas de nervios; semillas dispuestas en una sola hilera. Cardamine

10b. Silícuas con nervios en la superficie; semillas dispuestas en dos hileras.

Nasturtium

11a. Plantas con hojas y tallos carnosos; silícuas de gran tamaño sin septum y con nervios bien marcados en la superficie.

Aschersoniodoxa

11b. Plantas no carnosas; silicuas pequeñas con septum y sin nervios en la superficie.

12a. Hierbas o sufrútices con tallos erguidos.

12b. Hierbas cespitosas de escaso tamaño con tallos generalmente postrados.

13a. Hierbas puberulentas con hojas papiráceas y silícuas cilíndricas.

Halimolobus 13b. Hierbas pubescentes con hojas no papiráceas y silícuas aciculares.

Sisymbrium

14a. Hojas sésiles; flores solitarias.

14b. Hojas basales pecioladas; flores agrupadas en racimos simples.

15a. Hojas canescentes con lámina ancha; flores sobre pedicelos cortos.

Englerocharis

15b. Hojas no canescentes con lámina estrecha y pequeña; flores sobre pedicelos bastante alargados.

Brayopsis

16a. Plantas poco ramificadas; hojas dispuestas en roseta.

Catadysia

16b. Plantas bastante ramificadas; hojas no dispuestas en roseta.

Weberbauera

\section{Aschersoniodoxa Gilg \& Muschl.}

Género sudamericano compuesto por tres especies, dos de las cuales se encuentran en el Perú.

\section{Aschersoniodoxa cachensis (Speg.) Al-Shehbaz}

Hierba suculenta poco ramificada de hasta $30 \mathrm{~cm}$ de alto. Hojas espatuladas dispuestas en rosetas en el extremo distal de las ramas. Flores grandes de pétalos blancos o amarillentos. Frutos grandes y anchos con nervaduras en la superficie exterior y sin septum.

Se desarrolla en suelos crioturbados en poblaciones con escaso número de individuos.

Material examinado: Aija: Abra de Huancapeti, 4500 - 4650 m, 25 mar. 2002, A. Cano et al. 12150 (USM).

\section{Brassica L.}

Género de origen eurasiático, con 30 especies a nivel mundial, algunas de las cuales son cultivadas o cosmopolitas. En el Perú se encuentran registradas cuatro especies.

\section{Clave para determinar las especies del género Brassica :}

1a. Silicuas de hasta $2 \mathrm{~cm}$ de largo. B. nigra

1b. Silicuas de más de $3 \mathrm{~cm}$ de largo.

2a. Hojas superiores con base auriculadasemiabrazadora; hojas inferiores lirado-pinnatífidas con lóbulos laterales anchos y grandes; flores en racimos en los que las flores maduras superan en longitud a los botones florales; silicuas de 5-6 cm de largo.

B. rapa subsp. campestris 
2b. Hojas superiores sésiles pero con base no auriculada o semiabrazadora; hojas inferiores lirado-pinnatífidas con lóbulos laterales angostos y pequeños; flores en racimos en los que los botones florales superan en longitud a las flores maduras; silicuas de 3-5 cm de largo.

B.juncea

\section{Brassica juncea (L.) Cosson}

Hierba erguida de tallos angulados y glaucos. Hojas inferiores lirado-pinnatífidas y pecioladas; hojas superiores generalmente sésiles y lanceoladas. Flores amarillas. Silicua glabra con semillas de color marrón oscuro.

Se la encuentra como especie invasora en áreas alteradas por actividad del hombre y zonas de cultivo sobre suelo húmedo y arcilloso.

Material estudiado: Huaylas: Pueblo Libre, $4100-4510$ m, 9 ago. 2001, C. Monsalve y A. Cano 8b (USM).

Nombre vulgar: «Mostaza».

\section{Brassica nigra (L.) Koch}

Hierba erguida de hasta 1,80 m de alto, con tallos cilíndricos, híspidos o glabros. Hojas inferiores lirado-pinnatífidas; hojas superiores lanceolado-elípticas. Flores amarillas. Silicua con nervadura central marcada y semillas de color castaño rojizo.

Se la encuentra como especie invasora en áreas alteradas por actividad del hombre y zonas de cultivo.

Material estudiado: Huaylas: Pueblo Libre, 4100 - 4510 m, 9 ago. 2001, C. Monsalve y A. Cano 8c (USM); Cerro Yanaico, 2400 - $2600 \mathrm{~m}$, 12 abr. 2001, J. Roque et al. 2591 (USM).

Nombre vulgar: «Mostaza negra».

\section{Brassica rapa subsp. campestris (L.) Clapham}

Hierba erguida de tallos cilíndricos generalmente glaucos. Hojas inferiores lirado-pinnatífidas; hojas superiores oblongo-lanceoladas. Flores grandes y amarillas. Silicua glabra con semillas redondeadas de color castaño oscuro

Se la encuentra como especie invasora en áreas alteradas por actividad del hombre y zonas de cultivo.
Material estudiado: Huaylas: Cerro Yanaico, Pueblo Libre, 2400 - 2600 m, 12 abr. 2001, C. Monsalve y A. Cano 2 (USM); Cerro Yanaico, Pueblo Libre, 2400 - 2600 m, 12 abr. 2001, J. Roque et al. 2578 (USM); Pamparomás, 2770 m, J. Albán 11299 (USM).

Nombre vulgar: «Nabo».

\section{Brayopsis Gilg \& Muschl.}

Género principalmente andino conformado por 14 especies de las cuales, tres están registradas para el Perú.

\section{Clave para determinar las especies del género Brayopsis :}

1a. Hojas escasamente pubescentes y ovario glabro; pétalos y sépalos subiguales. B. calycina

1b. Hojas y ovario densamente pubescentes; pétalos notablemente más grandes que los sépalos.

B. alpaminae subsp. smithii

\section{Brayopsis alpaminae subsp. smithii}

\section{Al-Shehbaz}

Hierba pulviniforme de tallo corto. Hojas ovado-lanceoladas densamente pubescentes con tricomas simples y largos. Flores solitarias. Silicua elipsoidal y pubescente, con semillas de color castaño oscuro.

Se la encuentra de manera frecuente en pajonales de puna.

Material estudiado: Huaylas: Ocshapampa, Pamparomás, 4350 - 4630 m, 11 oct. 1999, C. Monsalve y A. Cano 4 (USM).

\section{Brayopsis calycina Gilg \& Muschl.}

Hierba cespitosa de tallo corto cubierto por los restos de las bases de las hojas. Hojas ovadolanceolada con tricomas rígidos. Flores solitarias. Silicua con semillas color castaño oscuro.

Se la encuentra de manera frecuente en pajonales de puna.

Material estudiado: Aija: Abra de Huancapeti, 4500 - 4650 m, 25 mar. 2002, A. Cano et al. 12095 (USM). Asunción: Chacas, abra de Punta Olímpica, 24 abr. 2004, A. Cano et al. 14503 (USM). Huari: P. N. Huascarán, 
Tunel Cahuish, 4500 - 4550 m, 10 jul. 1985, D. N. Smith 11116 (MO, USM); P. N. Huascarán, Tunel Cahuish, 4400 - 4450 m, 11 jun. 2002,A. Cano et al. 12386 (USM). Huaylas: Abra de Tres Cruces, 4400-4600 m, 18 may. 2000, A. Cano et al. 10470 (USM); Quebrada Cachicoral, Pamparomás, 3900 - 4400 m, 12 oct. 1999, A. Cano et al. 9794 (USM); Cerro Rico, Pamparomás, 4400 - 4600 m, 28 mar. 2002, C. Monsalve y A. Cano 19 (USM); Cerro Rico, Pamparomás, 4400 - 4600 m, 28 mar. 2002, C. Monsalve y A. Cano 20 (USM); Cerro Rico, Pamparomás, 4400 - 4600 m, 28 mar. 2002, C. Monsalve y A. Cano 21 (USM); Riurín, 42004350 m, 18 may. 1999, A. Cano et al.9139 (USM); P. N. Huascarán, Quebrada Alpamayo, 4600-4850 m, D. N. Smith 9737 (MO, USM); P. N. Huascarán, Quebrada Los Cedros, 46004850 m, D. N. Smith 9952 (MO, USM).

\section{Capsella Medik.}

Género que comprende cinco especies distribuidas principalmente en regiones templadas a cálidas. En el Perú sólo se registra una especie de distribución cosmopolita.

\section{Capsella bursa-pastoris (L.) Medik.}

Hierba erguida y pubescente con tallos cilíndricos, ramificados desde la base. Hojas basales arrosetadas, enteras o pinnatisectas, pecioladas; hojas superiores lanceoladas, sésiles y sagitadas en la base. Flores blancas o rosadas dispuestas en racimos corimbiformes. Silícula, obdeltoidea, cordada, con semillas de color castaño claro.

Se la encuentra con frecuencia en áreas alteradas por actividad del hombre o eventualmente entre arbustos o pastizales que crecen sobre las laderas rocosas.

Material estudiado: Aija: La Merced, 3200 -3500 m, 25 mar. 2002, A. Cano et al. 12164 (USM). Bolognesi: Pocpa, 3350 - 3700 m, 18 jul. 1998, A. Cano et al. 8518 (USM). Huari: Juprog, Campamento Minero Antamina, 3800 m, 17 dic. 2002, A. Cano et al. 12823. Huaylas: Huashta Cruz, Pueblo Libre, 4000-4100 m, 29 Mar. 2002, C.
Monsalve y A. Cano 26 (USM); Pamparomás, 2900 m, J. Albán 11465 (USM); Pamparomás, 2770 m, J. Albán 11589 (USM); Pamparomás, J. Albán 13693 (USM).

Nombre vulgar: «Bolsa del Pastor».

\section{Cardamine L.}

Comprende alrededor de 130 especies distribuidas en regiones templadas. En el Perú se encuentran presentes cuatro especies.

8. Cardamine bonariensis Pers.

Hierba rizomatosa con tallos decumbentes y raíces adventicias en los nudos. Hojas pinnatipartidas, pecioladas, base auriculada. Flores blancas. Silicua glabra con semillas en números de 20 ó 30 por fruto dispuestas en dos series.

Se la encuentra generalmente como planta emergente enraizada en acequias y pequeños arroyos.

Material estudiado: Carhuaz: P. N. Huascarán, Quebrada Ishinca, 4400 m, 13 feb. 1985, D. N. Smith 9542 (MO, USM). Huaráz: P. N. Huascarán, Quebrada Shallap, 3900 m, 20 feb. 1985, D. N. Smith 9676 (MO, USM). Huaylas: Pueblo Libre, 4100 - 4510 m, 9 ago. 2001, C. Monsalve y A. Cano 9 (USM); P. N. Huascarán, Quebrada los Cedros, 4020 - 4050 m, 21 feb. 1985, D. N. Smith 9836 (MO, USM). Recuay: Marca, 2600 m, 9 ago. 1963, J. Gómez 77 (USM); P. N. Huascarán, Río Pachacoto, 4200 m, 17 ene. 1985, D. N. Smith 9383 (MO, USM). Yungay: P. N. Huascarán, Quebrada Ranincuray, 4000 - 4300 m, 12 feb. 1985, D. N. Smith 9109 (MO, USM); P. N. Huascarán, Quebrada Ranincuray, 4100 - 4200 m, 18 abr. 1985, D. N. Smith 10413 (MO, USM).

Nombre vulgar: «Berro».

\section{Catadysia O. E. Schulz}

Género monotípico endémico del Perú.

\section{Catadysia rosulans O. E. Schulz}

Hierba cespitosa con ramas postradas y dispuestas en rosetas. Hojas espatuladas, pecioladas, de color violáceo. Flores con pétalos blancos o lilas, espatulados, emarginados. 
Silícuas pequeñas, anchas, ovoides, con nervación reticulada.

Especie muy poco frecuente, endémica del departamento de Ancash.

Material estudiado: Santa: Macate, 4140-4620 m, 21 may 2000, A. Cano et al. 10557 (USM).

\section{Cremolobus DC.}

Género típicamente andino, con siete especies conocidas, de las cuales cinco se encuentran en el Perú y una es considerada endémica.

\section{Cremolobus chilensis DC.}

Hierba erguida, hasta de $50 \mathrm{~cm}$ de alto. Hojas generalmente alternas y de morfología variada. Flores blancas con ginóforo. Silícula con valvas generalmente orbiculares, erguidas o pendientes del septo transversal y con una sola semilla por valva. Generalmente se la encuentra en matorrales sobre laderas rocosas de pendiente no muy pronunciada.

\section{Material estudiado: Bolognesi:} Capillapunta, 3540 m, 14 abr. 1949, R. Ferreyra 5718 (USM); Jerusalem, 3200 - 3500 m, 18 may. 1950, R. Ferreyra 7520 (MO, USM). Casma: Lomas de Casma, 400 - 500 m, 9 set. 1950, R. Ferreyra 8060 (USM). Corongo: Tarica, 24 may. 2000, J. Roque et al. 1601 (USM). Huari: P. N. Huascarán, Tunel Cahuish, 4350 m, 31 mar. 1985, D. N. Smith 10168 (MO, USM). Huaylas: Cerro Yanaico, Pueblo Libre, 2350-2800 m, 16 may. 1999, A. Cano 8976 (USM); Pamparomás, 2750 m, J. Albán 12853 (USM).

Descurainia Webb. \& Benth.

Este género agrupa cerca de 55 especies. Está principalmente distribuido en las regiones templadas y frías del hemisferio norte y sur de África. Para la flora peruana se ha registrado cinco especies.

\section{Clave para determinar las especies del género Descurainia :}

1a. Silicuas agrupadas en densos racimos corimbiformes. D. athrocarpa

1b. Silicuas agrupadas en racimos alargados. 2a. Silicuas de 4-10 mm de largo.

D. depressa

2b. Silicuas de 10-19 mm de largo.

3a. Hojas con 8 a 10 pares de pinnas. D. myriophylla

3b. Hojas con 4 a 6 pares de pinnas. D. leptoclada

\section{Descurainia athrocarpa (Gray) O. E.} Schulz

Hierba erguida, pubescente y de tallos cilíndricos. Hojas bipinnatífidas. Flores amarillentas dispuestas en densos racimos corimbiformes. Silicua con semillas de color anaranjado claro dispuestas en una serie.

Se la encuentra preferentemente sobre laderas rocosas en poblaciones no muy numerosas.

Material estudiado: Carhuaz: P. N. Huascarán, Quebrada Ishinca, 4730 - 4930 m, 12 feb. 1985, D. N. Smith 9450 (MO, USM). Huari: Yanacancha, $4700-4820 \mathrm{~m}$, 7 ago. 2003, A. Cano et al. 13685 (USM). Huaylas: Huashta Cruz, 4000-4200 m, 16 may. 2000, A. Cano et al. 10221 (USM); Ocshapampa, Pamparomás, 4350-4630 m, 11 oct. 1999, A. Cano et al. 9767 (USM); P. N. Huascarán, Quebrada Alpamayo, 4020 - 4250 m, 1 abr. 1985, D. N. Smith 9827 (MO, USM); P. N. Huascarán, Quebrada los Cedros, 40204050 m, 1 abr. 1985, D. N. Smith 9827 (USM); Riurín, 4300-4620 m, 19 may. 1999, A. Cano et al. 9239 (USM). Recuay: P. N. Huascarán, Quebrada Quenua Ragra, 4600 - 4700 m, 10 may. 1985, D. N. Smith 10637 (MO, USM); P. N. Huascarán, Quebrada Quenua Ragra, 4700 - 4850 m, 11 mar. 1986, D. N. Smith 11746 (MO, USM); P. N. Huascarán, Río Pachacoto, 4620 - 4720, 28 may. 2001, A. Cano et al. 11434, 11500 (USM). Santa: Macate, 3100 - 3310 m, 20 may. 2000, J. Roque et al. 1393 (USM).

Nombre vulgar: «Alcalde-ccora».

12. Descurainia depressa (Phil.) Reiche

Hierba pubescente con tallos postrados. Hojas bipinnatífidas. Flores blancas o amarillentas, pequeñas. Silicua con semillas de color anaranjado claro dispuestas en una serie. 
Se la encuentra preferentemente sobre laderas rocosas en poblaciones no muy numerosas.

Material estudiado: Huaylas: Cerro Rico, Pueblo Libre, 4400-4600 m, 28 mar. 2002, C. Monsalve y A. Cano 25 (USM).

\section{Descurainia leptoclada Muschl.}

Hierba pubescente de tallos ramificados desde la base. Hojas bipinnatífidas. Flores amarillas. Silicua glabra con semillas dispuestas en una sola serie.

Se la encuentra en gran número sobre laderas rocosas.

Material estudiado: Huaylas: Cerro Rico, Pamparomás, 4400 - 4600 m, 28 mar. 2002, C. Monsalve y A. Cano10 (USM); Cerro Rico, Pamparomás, 4400 - 4600 m, 28 mar 2002, C. Monsalve y A. Cano 11 (USM); Huashta Cruz, Pueblo Libre, 4000 - 4200 m, 16 may. 2000, A. Cano et al. 10221 (USM); Riurín, 4300 - 4620 m, 19 may. 1999, A. Cano et al. 9245 (USM).

\section{Descurainia myriophylla (Willd.)} Fries

Hierba pubescente con tallos cilíndricos. Hojas grandes, bipinnatífidas. Flores amarillas. Silicua glabra con semillas de color castaño claro dispuestas en una sola serie.

Se la encuentra preferentemente sobre laderas rocosas en poblaciones no muy numerosas entre arbustos.

Material estudiado: Aija: Aija, 3890-4150 m, 28 may. 2001, A. Cano et al. 11634 (USM). Bolognesi: Chiquián, 3350 m, 13 may. 1950, E. Cerrate 606 (USM). Huaraz: P. N. Huascarán, Quebrada Shallap, 3700 - 4000 m, 6 mar. 1986, D. N. Smith 10738 (MO, USM). Huaylas: Pamparomás, 2330 m, J. Albán 11394 (USM); Pamparomás, 2330 m, J. Albán 11634 (USM); Pamparomás, 2330 m, J. Albán 13729 (USM); P. N. Huascarán, Auquispuquio, 3900 m, 6 abr. 1986, D. N. Smith 11904 (MO, USM); Riurín, 4300 - 4620 m, 19 may. 1999, A. Cano et al. 9226 (USM). Santa: Macate, 4140 - 4620 m, 21 may. 2000, A. Cano et al. 10568 (USM).
Nombre vulgar: «Hierba de San Juan de Dios».

\section{Draba L.}

Es el género más diverso de la familia con alrededor de 300 especies distribuidas en regiones templadas y boreales. En el Perú está representado por 18 especies, de las cuales 9 son endémicas.

\section{Clave para determinar las especies del género Draba :}

1a. Tallos escaposos; plantas cespitosas o pulviniformes.

1b. Tallos no escaposos; plantas generalmente sufrutescentes.

2a. Escapos notablemente largos incluso en flor. (3)

2b. Escapos bastante cortos incluso en fruto.

3a. Silícuas glabras o glabrescentes.

D. macleanii

3b. Silícuas densamente pubescentes

4a. Flores con pétalos blancos de $4 \mathrm{~mm}$ de largo; silícuas en racimos densos.

D. pickeringii

4b. Flores con pétalos amarillos menos de $4 \mathrm{~mm}$ de largo; silícuas en racimos laxos. D. brackenridgei

5a. Flores con pétalos amarillos; silícuas agrupadas en racimos corimbiformes.

D. alchemilloides

$5 b$. Flores con pétalos blancos; silícuas agrupadas en racimos simples

6a. Plantas de hasta $20 \mathrm{~cm}$ de alto, bastante ramificadas; hojas lenticulares densamente agrupadas; flores con pétalos más cortos que los sépalos.

D. depressa

6b. Plantas enanas de hasta $5 \mathrm{~cm}$ de alto; hojas no lenticulares; flores con pétalos y sépalos de igual tamaño.

D. cryptantha

7a. Plantas de hasta $30 \mathrm{~cm}$ de alto, bastante ramificadas; flores grandes con pétalos amarillos.

\section{D. ochropetala}

7b. Plantas pequeñas de hasta $15 \mathrm{~cm}$ de alto; flores con pétalos blancos

8a. Silícua ovado-elíptica, pubescente. D. alyssoides

8b. Silícua ovado-lanceolada, glabra.

D. hemsleyana

\section{Draba alchemilloides Gilg}

Hierba pulviniforme, densamente pubescente, flocosa. Hojas dispuestas en rosetas 
hacia los extremos de las ramas, obovadas, de aspecto canescente. Flores con pétalos amarillos. Silícuas ovoides, pubescentes, agrupadas en racimos corimbiformes.

Especie no muy frecuente, anteriormente considerada endémica de Ancash pero actualmente se sabe que su distribución se extiende hacia el norte hasta Ecuador.

Material examinado: Carhuaz: P. N. Huascarán, Quebrada Ishinca, 4730 m, 12 feb. 1985, D. N. Smith 9451 (HUT, MO). Huaraz: P. N. Huascarán, Quebrada Llaca, 4500 m, 5 ene. 1985, D. N. Smith 8962 (HUT, MO).

\section{Draba alyssoides Humb. \& Bonpl. ex} DC.

Hierba erguida e hirsuta de hasta $20 \mathrm{~cm}$ de alto. Hojas oblongo-lanceoladas, sésiles y con uno o dos dientes a cada lado de la lámina. Flores blancas. Silicua ovada con tricomas simples y semillas de color marrón oscuro dispuestas en dos hileras.

Se la encuentra de manera dispersa y escasa en laderas rocosas a la sombra de los arbustos.

Material estudiado: Carhuaz: Ponquez, 2800 - 3600 m, 16 abr. 2001, A. Cano et al. 11229 (USM). Huari: Yanacancha, 4650 - 4750 m, 5 ago. 2003, A. Cano et al. 13656 (USM). Huaylas: Cerro Yanaico, Pueblo Libre, 2400 - 2650 m, 12 abr. 2001, A. Cano et al. 11040 (USM).

\section{Draba brackenridgei Gray}

Hierba pequeña, pubescente y cespitosa con tallo escaposo. Hojas oblongas, dispuestas en rosetas alrededor del tallo. Flores blancas dispuestas en racimos corimbosos. Silicua ovado-lanceolada con tricomas bifurcados y semillas alargadas de color marrón oscuro dispuestas en dos hileras.

Se la encuentra en laderas rocosas de alta montaña en poblaciones con escasos individuos.

Material estudiado: Huaylas: Riurín, 4200 - 4350 m, 18 may. 1999, A. Cano et al. 9131 (USM); Riurín, 4200 - 4350 m, 18 may. 1999, A. Cano et al. 9137 (USM).

\section{Draba cryptantha Hook.}

Hierba pulviniforme y pubescente con tallos escaposos cortos. Hojas obovadas, dispuestas en densas rosetas. Flores blancas dispuestas en racimos corimbiformes. Silicua pequeña, ovoide, con tricomas simples y semillas de color castaño oscuro.

Se la encuentra en laderas rocosas de alta montaña, en poblaciones con escasos individuos.

Material estudiado: Huaylas: Abra Tres Cruces, 4400 - 4600 m, 18 may. 2000,A. Cano et al. 10456(USM); Ocshapampa, Pamparomás, 4350 - 4630 m, 11 oct. 1999, A. Cano et al. 9767 (USM). Recuay: P. N. Huascarán, Quenua Ragra, 4600 - 4700 m, 10 may. 1985, D. N. Smith 10673 (MO, USM); P. N. Huascarán, Río Pachacoto, 4620 - 4720 m, 28 may. 2001, A. Cano et al. 11459, 11467 (USM).

\section{Draba depressa Hook.}

Hierba erguida pulviniforme. Hojas obovadas, muy pequeñas, lenticulares, dispuestas en rosetas. Flores blancas en racimos cortos que a penas sobresalen de las ramas. Silícuas pequeñas, ovoides, pubescentes.

Se la encuentra en laderas rocosas de alta montaña, en poblaciones con escasos individuos.

Material estudiado: Asunción: Chacas, abra de Punta Olímpica, 24 abr. 2004, A. Cano et al. 14512 (USM). Huaylas: P. N. Huascarán, Quebrada Los Cedros, 2600 2850 m, 12 mar. 1985, D. N. Smith 9951 (MO, USM). Yungay: P. N. Huascarán, Quebrada Ranincuray, 4700 m, 15 ene. 1985, D. N. Smith 9231 (MO, USM).

\section{Draba hemsleyana Gilg.}

Hierba erguida y pubescente con tallo ramificado. Hojas inferiores oblongas; hojas superiores más pequeñas, linear-oblongas. Flores blancas. Silícua ovado-lanceolada, glabra y con semillas dispuestas en dos hileras.

Se la encuentra en laderas rocosas de alta montaña en poblaciones con escasos individuos.

Material estudiado: Huaylas: Laguna 
Parón, Caraz, 4100 - 4400 m, 8 dic. 1999, M. I. La Torre et al. 2633 (USM).

\section{Draba macleanii Hook.}

Hierba cespitosa de escaso tamaño. Hojas oblongas u obovadas dispuestas en roseta en la base de los tallos. Flores blancas agrupadas en racimos de 12 a 15 flores. Silícuas glabras, elípticas.

Frecuente en laderas rocosas con poco número de individuos.

Material estudiado: Carhuaz: P. N. Huascarán, Quebrada Quenua Ragra, Parque Nacional Huascarán, 4730 - 4930 m, 12 feb. 1985, D. N. Smith 9451 (MO, USM). Huaraz: P. N. Huascarán, Quebrada Shallap, 4400 4600 m, 24 may 1985, D. N. Smith 10823 (MO, USM). Recuay: P. N. Huascarán, Quebrada Ishinca, 4500 - 4600 m, 11 mar. 1986, D. N. Smith, 11738 (MO, USM).

\section{Draba ochropetala O. E. Schulz}

Sufrútice de hasta $30 \mathrm{~cm}$ de alto, bastante ramificado y densamente pubescente, cinéreo, canescente. Hojas oblongas u ovadas, con bordes levemente dentados. Flores con pétalos amarillos dispuestos en racimos de hasta 15 flores. Silícuas ovoides, densamente pubescentes con tricomas simples.

Especie endémica del departamento de Ancash. El ejemplar tipo fue colectado por Weberbauer en al Cordillera de Pelagatos, provincia de Pallasca. Esta especie se desarrolla en laderas rocosas, secas.

Material estudiado: Huari: Juprog, campamento minero Antamina, $3800 \mathrm{~m}, 17$ dic. 2002,A. Cano et al. 12854 (USM).Yanacancha, 4500 - 4600 m, 12 may. 2003, A. Cano et al. 13129 (USM); Yanacancha, 4150-4750 m, 6 ago. 2003,A. Cano et al. 13664 (USM).

\section{Draba pickeringii Gray}

Hierba cespitosa, pubescente y de tallo escaposo. Hojas oblongas. Flores blancas dispuestas en racimos corimbosos. silicua ovoide, pubescente y semillas redondeadas de color marrón oscuro, dispuestas en dos series.
Se la encuentra en laderas rocosas de alta montaña, en poblaciones con escasos individuos.

Material estudiado: Aija: Abra de Huancapeti, 4490 - 4560 m, 29 may. 2001, B. León et al. 4978 (USM). Antonio Raimondi: Laguna Yanacocha, 4200 - 4300 m, 22 ago. 2003, A. Cano et al. 13731 (USM). Huaráz: P. N. Huascarán, Quebrada Llaca, 4500 m, 5 ene. 1985, D. N. Smith 8962 (MO, USM). Huari: Juprog, campamento minero Antamina, 3800 m, 17 dic. 2002, A. Cano et al. 12806 (USM). Huaylas: Cerro Rico, Pueblo Libre, 4400 - 4600 m, 28 mar. 2002, C. Monsalve y A. Cano12 (USM); Cerro Rico, Pueblo Libre, 4400 - 4600 m, 28 mar. 2002, C. Monsalve y A. Cano 13 (USM); Cerro Rico, Pueblo Libre 4400-4600 m, 28 mar. 2002, C. Monsalve y A. Cano 14 (USM); Laguna Parón, Caráz, 4100 - 4400 m, 8 dic. 1999, M. I. La Torre et al. 2615 (USM).

\section{Englerocharis Muschl.}

Género altoandino conformado por tres especies, todas presentes en el Perú, y de las cuales una es endémica.

\section{Englerocharis peruviana Muschl.}

Hierba cespitosa de escaso tamaño, densamente pubescente con tricomas alargados simples que le dan un color cinéreo, plateado. Hojas oblongas u obovadas, sésiles, dispuestas en rosetas. Flores blancas. Silícuas elipsoidales, glabras, agrupadas en racimos cortos en números de hasta 10 silícuas por racimo.

Especie poco frecuente que se desarrolla en suelos crioturbados a grandes altitudes.

Material estudiado: Huari: San Marcos, 4700 - 4800 m, 26 mar. 2004, A. Cano et al. 14165 (USM).

\section{Halimolobus Wittstein}

Género conformado por especies de distribución tropical, dos de las cuales se encuentran en el Perú.

\section{Halimolobus hispidula (DC.) O. E. Schulz}

Hierba erguida de hasta $30 \mathrm{~cm}$ de alto, poco ramificada, pubescente. Hojas con láminas 
membranáceas, muy delgadas, con bordes ondeados, crenados. Flores blancas dispuestas en racimos bastante alargados y en números de 30 a 40 flores por racimos. Silícuas alargadas, cilíndricas, pubescentes. Semilllas de consistencia mucilaginosa cuando húmedas.

Se desarrolla a altitudes medias en ambientes húmedos.

Material estudiado: Carhuaz: Ponquez, 2800 - 3600 m, 16 mar. 2001, Cano, A. 11227 , 11232 (USM).

\section{Lepidium L.}

Este género agrupa cerca de 150 especies. Tiene una distribución cosmopolita, y es especialmente importante en las regiones templadas. En la flora peruana están representadas 14 especies.

\section{Clave para determinar las especies del género Lepidium :}

1a. Silículas romboidales con ápice cerrado, no emarginado

1b. Silículas circulares u ovado-elipsoidales con ápice abierto, claramente emarginado

2a. Estilos alargados, exertos de hasta $1 \mathrm{~mm}$ de largo; plantas usualmente perennes, con raíces gruesas.

\section{L. meyenii}

2b. Estilos cortos y en ocasiones ausentes; plantas anuales de porte herbáceo.

L. kalenbornii

3a. Hojas con lámina entera, bordes irregulares serrado-dentados y bases no auriculadas.

\section{L. chichicara}

3b. Hojas con lámina hendida o partida y bases generalmente auriculadas

4a. Hojas bipinnatífidas con base siempre auriculada.

L. bipinnatifidum

4b. Hojas pinnatífidas con base en ocasiones no auriculada

5a. Silículas densamente pubescentes; pedicelos aplanados con márgenes alados. L. pubescens

5 b. Silículas glabras; pedicelos no aplanados sin márgenes alados.

L. virginicum

\section{Lepidium bipinnatifidum Desv.}

Hierba de tallos postrados dispuestos en roseta. Hojas inferiores bipinnatífidas; hojas superiores binnatipartidas o enteras. Flores blancas en pedúnculos florales recurvados. Silícula ovado-elíptica, con ápice notablemente emarginado y una semilla de color anaranjado claro por valva.

Se la encuentra con frecuencia en áreas alteradas por actividad del hombre, al borde del camino o eventualmente entre arbustos o pastizales que crecen sobre las laderas rocosas.

Material estudiado: Aija: La Merced, 3200 -3500 m, 25 mar. 2002, A. Cano et al. 12172 (USM). Bolognesi: Lihuán, 3870 - 3950 m, 18 jul. 1998, A. Cano et al. 8549 (USM). Carhuaz: Carhuáz, 3600 - 3650 m, 15 abr. 2001, A. Cano 11173 (USM); P. N. Huascarán, Quebrada Ishinca, 4370 m, 11 feb. 1985, D. N. Smith 9446 (MO, USM); P. N. Huascarán, Quebrada Ishinca, 4100 - 4250 m, 14 feb. 1985, D. N. Smith 9563 (MO, USM). Huaraz: P. N. Huascarán, Quebrada Shallap, $3690-$ 4100 m, 20 feb. 1985, D. N. Smith 9653 (MO, USM). Huaylas: Abra Tres Cruces, $4400-$ 4600 m, 18 may. 2000, A. Cano et al. 10396 (USM); Cerro Rico, Pamparomás, 4400 - 4600 m, 28 mar. 2002, C. Monsalve y A. Cano 17 (USM); C. Rico, Pamparomás, 4400 - 4600 m, 28 mar. 2002, C. Monsalve y A. Cano 18 (USM); Cerro Yanaico, Pueblo Libre; 24002600 m, 12 abr. 2001, C. Monsalve y A. Cano 6 (USM); Cerro Yanaico, Pueblo Libre, 24002600 m, 12 abr. 2001, J. Roque et al. 2643a (USM); Cerro Yanaico, Pueblo Libre, 24002600 m, 12 abr. 2001, J. Roque et al. 2643b (USM); Huashta Cruz, Pueblo Libre, 4000 4200 m, 16 may. 2000, A. Cano et al.10225 (USM); La Hoyada, Pueblo Libre, 3100 - 3400 m, 17 May. 2000, A. Cano et al. 10396 (USM); Pamparomás, 4350 m, J. Albán 11608 (USM); Pamparomás, 2650 m, J. Albán 12910 (USM); Putaca, 3100 m, J. Albán 12938 (USM); P. N. Huascarán, Auquispuquio, 3750 - 3900 m, D. N. Smith 12004 (MO, USM); P. N. Huascarán, Quebrada Santa Cruz, 4300 - 4800 m, 16 ene. 1985, D. N. Smith 9292 (MO, USM); P. N. Huascarán, Quebrada Santa Cruz, 14 oct. 1999, A. Cano et al. 9879 (USM). Yungay: 
Yauya, $3500-3800$ m, 10 jul. 1982, A. Gentry 37382 (MO, USM).

Nombre vulgar: «Mostacilla».

\section{Lepidium chichicara Desv.}

Hierba erguida de tallos ramificados y acanalados. Hojas linear-oblongas y sésiles. Flores blancas. Silícula con una semilla de color anaranjado claro por valva.

Se la encuentra con frecuencia en áreas alteradas por actividad del hombre o eventualmente entre arbustos que crecen sobre las laderas rocosas.

Material estudiado: Huaylas: Huaylas, 4200 m, E. Carrillo 1243 (USM); Pueblo Libre, 2300 m, 12 abr. 2001, C. Monsalve y A. Cano 1a (USM); Cerro Yanaico, Pueblo Libre, 2400 - 2600 m, 12 abr. 2001, C. Monsalve y A. Cano 7 (USM); Pamparomás, 3200 m, J. Albán (s/n); Pamparomás, 4350 m, J. Albán (s/n); P. N. Huascarán, Quebrada Santa Cruz, $2700-3550$ m, 14 oct. 1999, A. Cano et al. 9879 (USM). Yungay: P. N. Huascarán, Sector Llanganuco, 3580 - 3850 m, 30 dic. 1984, D. N. Smith 8839 (MO, USM).

Nombre vulgar: «Chichicara».

\section{Lepidium kalenbornii Hitchc.}

Hierba erguida, glabrescente. Hojas inferiores pinnatífidas y superiores pinnatipartidas. Flores blancas. Silícula romboidal con estilo muy corto y en ocasiones ausente.

Especie endémica de los Andes del centro y sur del Perú.

Material estudiado: Recuay: P. N. Huascarán, Quebrada Quenua Ragra, 4500 4600 m, 3 mar. 1986, D. N. Smith 11732B (MO, USM).

\section{Lepidium meyenii Walp.}

Hierba de tallos usualmente postrados y raíz gruesa. Hojas inferiores pinnatífidas y superiores pinnatipartidas. Flores blancas. Silícula romboidal con estilo alargado y exerto.

Se desarrolla en ecosistemas altoandinos en la sierra central y sur del Perú.
Material estudiado: Recuay: P. N. Huascarán, Quebrada Quenua Ragra, 4500 4600 m, 3 mar. 1986, D. N. Smith 11732A (MO, USM).

Nombre vulgar: «Maca».

30. Lepidium pubescens Desv.

Hierba erguida, escasamente pubescente y con tallos angulados. Hojas inferiores pinnatífidas; hojas superiores pinnatipartidas. Flores blancas. Silícula ovado-elipsoidal con una semilla de color anaranjado claro por valva.

Se la encuentra generalmente en áreas alteradas por actividad del hombre.

Material estudiado: Huaylas: Santo Toribio, E. Carrillo 1260 (USM).

\section{Lepidium virginicum $\mathrm{L}$.}

Hierba erguida, pubescente y de tallos angulados. Hojas lirado-pinnatífidas. Flores blancas. Silícula ovado-elíptica con una semilla alargada de color anaranjado oscuro por valva.

Se le encuentra generalmente en áreas alteradas por actividad del hombre.

Material estudiado: Huaylas: Pueblo Libre, 2300 m, 12 abr. 2001, C. Monsalve y A. Cano 1b (USM).

\section{Mancoa Wedd}

Género altoandino que está conformado por ocho especies de las cuales solamente una se encuentra presente en el Perú.

\section{Mancoa hispida Wedd.}

Hierba cespitosa de tallos postrados, pubescente, con tricomas alargados simples y rígidos. Hojas dispuestas en rosetas, dentadas o subpinnatífidas. Flores blancas. Silícuas elipsoidales, pubescentes.

Se desarrolla a grandes altitudes en poblaciones con pocos individuos. No es muy frecuente.

Material estudiado: Huari: Yanacancha, 4650 - 4750 m, 5 ago. 2003, A. Cano et al. 13635 (USM). Recuay: P. N. Huascarán, Río Pachacoto, 4620 - 4720 m, 28 may. 2001, A. 
Cano et al. 11434 (USM); P. N. Huascarán, Río Pachacoto, 4230 m, 16 mar. 1986, D. N. Smith 11810 (MO, USM).

Nasturtium R. Br.

Comprende alrededor de 70 especies de distribución principalmente cosmopolita. En el Perú se conocen siete especies.

\section{Nasturtium officinale $\mathrm{R}$. Br.}

Hierba rizomatosa con tallos ramificados y raíces adventicias en los nudos inferiores. Hojas pinnatisectas con lóbulos de morfología variada. Flores blancas. Silicua globosa, con nerviación reticulada y deprimida entre las semillas. Semilas mucilaginosas en estado húmedo, de color castaño oscuro y superficie reticulada, dispuestas en dos series.

Se la encuentra generalmente como planta emergente enraizada en acequias y pequeños arroyos.

Material estudiado: Aija: Aija, 3890 - 4150 m, 28 may. 2001, A. Cano et al. 11563 (USM). Bolognesi: Pallca, 3700 m, 19 jul. 1998, A. Cano et al. 8566 (USM). Carhuaz: Ponquez, $2800-3600$ m, 16 abr. 2001, A. Cano et al. 11230 (USM). Carlos F. Fitzcarrald: Laguna Huachucocha, 4200 m, 23 jun. 2004, A. Cano et al. 14645 (USM). Huaylas: Cerro Yanaico, Pueblo Libre, 2400-2600 m, 12 abr 2001, C. Monsalve y A. Cano 5 (USM); Pueblo Libre, 2300 m, 12 abr. 2001, C. Monsalve y A. Cano 3 (USM).

Nombre vulgar: «Berro».

\section{Raphanus L.}

Comprende ocho especies principalmente distribuidas en regiones boreales. En el Perú se encuentran registradas sólo dos especies.

\section{Clave para determinar las especies del género Raphanus :}

1a. Flores con pétalos blancos o lilas; silícuas delgadas, de hasta $5 \mathrm{~mm}$ de diámetro.

$R$. raphanistrum

1b. Flores con pétalos siempre lilas con nervaduras oscuras de color púrpura; silícuas gruesas, de hasta $10 \mathrm{~mm}$ de diámetro. R. sativus

\section{Raphanus raphanistrum L.}

Hierba erguida, glabra o pubescente, de tallos poco ramificados. Hojas inferiores liradopinnatipartidas; hojas superiores enteras, sésiles. Flores con pétalos blancos o lilas. Silícuas gruesas de hasta $10 \mathrm{~mm}$ de diámetro, indehiscente con nervaduras longitudinales.

Se le encuentra como especie invasora o cultivada.

Material estudiado: Aija: La Merced $3200-$ 3500 m, 25 mar. 2002, Cano, A. 12154 (USM).

Nombre vulgar: «Rábano».

\section{Raphanus sativus L.}

Hierba erguida, glabra o pubescente, de tallos poco ramificados. Hojas inferiores liradopinnatipartidas; hojas superiores enteras, sésiles. Flores lilas con nervaduras de color púrpura oscuro. Silicuas delgadas de hasta 5 $\mathrm{mm}$ de diámetro, indehiscente y semillas de color castaño.

Se la encuentra como especie invasora o cultivada.

Material estudiado: Huaylas: Cerro Yanaico, Pueblo Libre, 2400 - 2600 m, 12 abr. 2001, J. Roque et al. 2592 (USM); La Hoyada, Pueblo Libre, 2600 - 3050 m, 15 may. 1999, A. Cano et al. 8929 (USM); Pamparomás, 2350 m, J. Albán 11323 (USM); Pueblo Libre, 4100 - 4510 m, 9 ago. 2001, C. Monsalve y A. Cano 8a (USM).

Nombre vulgar: «Rábano silvestre».

\section{Sisymbrium L.}

Comprende alrededor de 80 especies, de distribución principalmente boreal. En el Perú se encuentran registradas 13 especies, de las cuales seis son endémicas.

\section{Clave para determinar las especies del género Sisymbrium :}

1a. Silícuas de 1 a $1.5 \mathrm{~cm}$ de largo; pétalos amarillos; hojas pinnatisectas.

S. officinale

1b. Silícuas de 1.5 a $3 \mathrm{~cm}$ de largo; pétalos blancos o rosados; hojas con bordes dentados.

S. peruvianum 
36. Sisymbrium officinale (L.) Scop.

Hierba erguida de hasta $40 \mathrm{~cm}$ de alto, bastante ramificada, pubescente. Hojas pinnatisectas, pediceladas. Flores con pétalos amarillos. Silícuas gruesas, cortas y pubescentes.

Se le encuentra a altitudes medias en ambientes húmedos y calientes.

Material estudiado: Recuay: Marca, 2600 m, 17 abr. 1963, J. Gómez 42 (USM).

\section{Sisymbrium peruvianum DC.}

Hierba erguida e hirsuta, con tallo muy ramificado. Hojas ovado-elípticas, con bordes dentados y pecioladas. Flores blancas. Silicua pubescente y recurvada, con semillas de color anaranjado dispuestas en una sola serie.

Se la encuentra preferentemente sobre laderas rocosas, en poblaciones no muy numerosas.

Material estudiado: Huaylas: Cerro Rico, Pamparomás, 4400 - 4600 m, 28 mar. 2002, C. Monsalve 23 (USM).

\section{Weberbauera Gilg \& Muschl.}

Género andino con siete especies conocidas para el Perú, de las cuales tres son endémicas.

\section{Clave para determinar las especies del género Weberbauera :}

1a. Flores con pétalos amarillos; silícuas con el septum incompleto. W. perforata

1b. Flores con pétalos blancos; silícuas con septum completo

2a. Silícuas lanceolado-elípticas, pequeñas, agrupadas en racimos en números no mayores de diez. W. smithii

2b. Silícuas alargadas, curvadas, grandes, agrupadas en largos racimos en números de hasta 30.

\section{W. spathulifolia}

\section{Weberbauera perforata Al-Shehbaz}

Hierba cespitosa formadora de densos cojines debido a la disposición arrosetada de sus hojas. Pubescente con tricomas alargados simples y rígidos que le dan un color plateado y textura serícea a las hojas. Flores amarillas dispuestas en racimos cortos con escaso número de flores. Silícuas ovadoelipsoidales con septum incompleto.
Especie endémica de la sierra del centro y norte del Perú restringida a unas pocas localidades.

Material estudiado: Huari: San Marcos, 4700 - 4800 m, 26 mar. 2004, A. Cano et al. 14165 (USM); Yanacancha, 4500 - $4600 \mathrm{~m}$, 13 may. 2003, A. Cano et al. 13168 (USM).

\section{Weberbauera smithii $\mathrm{Al}-\mathrm{Shehbaz}$}

Hierba cespitosa de escaso tamaño. Hojas oblongas u obovadas, pecioladas. Flores blancas. Silícuas lanceolado-elípticas, glabras, con estilo alargado.

Especie endémica de la sierra del centro y norte del Perú restringida a unas pocas localidades.

Material estudiado: Yungay: P. N. Huascarán, Sector Llanganuco, $4770-4870 \mathrm{~m}$, 31 dic. 1984, D. N. Smith 8894 (tipo: MO, USM)

\section{Weberbauera spathulifolia (Gray)}

$$
\text { O. E. Schulz }
$$

Hierba postrada de tallos usualmente glabros. Hojas inferiores oblongas y pecioladas; hojas superiores pinnatisectas y sésiles. Flores blancas. Silicua glabra, con semillas de color castaño claro dispuestas en dos series.

Se la encuentra sobre laderas rocosas, formando poblaciones dispersas con bajo número de individuos.

Material estudiado: Bolognesi: Aquia, $4300-4430 \mathrm{~m}, 10$ jun. 2002, A. Cano et al. 12366 (USM). Huari: Juprog, campamento minero Antamina, 3800 m, 17 dic. 2002, A. Cano et al. 12807, 12808 (USM). Huaylas: Cerro Rico, Pamparomás, 4400 - 4600 m, 28 mar. 2002, C. Monsalve y A. Cano15 (USM); Cerro Rico, Pamparomás 4400 - 4600 m, 28 mar. 2002, C. Monsalve y A. Cano 22 (USM); Cerro Rico, Pamparomás 4400 - 4600 m, 28 mar. 2002, C. Monsalve y A. Cano 24 (USM); Huashta Cruz, Pueblo Libre, 4000 - 4200 m, 16 may. 2000, A. Cano et al. 10148 (USM); Huashta Cruz, Pueblo Libre, 4000 - 4200 m, 16 may. 2000, A. Cano et al. 10260 (USM); Riurín, 4300 - 4600 m, 19 may. 1999, A. Cano et al. 9238 (USM); P. N. Huascarán, Quebrada Alpamayo, 4020 - 4700 m, D. N. Smith 9924 
(MO, USM). Recuay: P. N. Huascarán, Pastoruri, 4700 - 4800 m, 31 mar. 1985, D. N. Smith 10182 (MO, USM); P. N. Huascarán, Quebrada Queshque, 4500 - 4600 m, 18 mar. 1986, D. N. Smith 11845 (MO, USM); P. N. Huascarán, Río Pachacoto, 4200 m, 17 ene. 1985, D. N. Smith 9373 (MO, USM); P. N. Huascarán, Runa Shimi, 4200-4300 m, 16 mar. 1986, D. N. Smith 11782A(MO, USM). Santa: Macate, 3100 - 3310 m, 20 may. 2000, J. Roque et al. 1485 (USM); Macate, 3100 - 3310 m, 20 may. 2000, J. Roque et al. 1529 (USM).

\section{Discusión y conclusión}

Con el presente trabajo se ha incrementado en siete el número de especies de la familia Brassicaceae presentes en Ancash de acuerdo a registros anteriores (Monsalve y Cano, 2003; Cano et al., 2000; Albán, 1998; Brako y Zarucchi, 1993; Smith, 1988; Cerrate, 1979). Estas adiciones incluyen a especies como Raphanus raphanistrum L. y Sisymbrium officinale (L.) Scop. cuya ocurrencia es relativamente frecuente debido a su comportamiento como malezas, pero que no fueron incluidas por Brako y Zarucchi (1993) para Ancash. Para el caso de las especies Draba depressa Hook y Draba macleanii Hook. estas fueron determinadas a partir de ejemplares almacenados en el Herbario San Mar$\cos$ (USM) y recolectados en años anteriores por D. N. Smith en el Parque Nacional Huascarán, pero que permanecían sin determinación y por lo tanto no fueron consideradas en su trabajo (Smith, 1988); mientras que para las especies Draba ochropetala Schulz, Halimolobus hispidula DC. y Weberbauera perforata Al-Shehbaz han sido confirmadas para Ancash con nuevas recolecciones y determinadas por los autores durante el presente trabajo. Es decir, el número de especies de Brassicaceae presentes en Ancash asciende a 45 con respecto a lo previamente reportado (Monsalve y Cano, 2003; Brako y Zarucchi, 1993).

A pesar de que se visitaron las localidades referidas en la literatura y además se realizaron colectas en localidades con característi- cas aparentemente similares, no se pudo confirmar la presencia de las especies Dactylocardamum imbricatifolium A1Shehbaz y Eudema incurva Al-Shehbaz que son reportadas por Brako y Zarucchi (1993) para Ancash y que además sólo se conocen de los ejemplares tipo. Es posible que estas especies presenten un nicho ecológico muy especializado, con características que no fueron especificadas por los colectores de los ejemplares tipo, y que por lo tanto hacen necesario continuar con una exploración botánica más exhaustiva en la zona.

También se presentan los nombres actualmente aceptados para cada una de las especies y que en algunos casos representan un cambio respecto al registro de Brako y Zarucchi (1993) como el nombre Rorippa nasturtium-aquaticum (L.) Hayek que actualmente se acepta como Nasturtium officinale $\mathrm{R} \mathrm{Br}$.

A diferencia de estudios anteriores (Monsalve y Cano, 2003; Smith, 1988), se han recolectado tres de las nueve especies consideradas como circunscritas en distribución a Ancash (Brako y Zarucchi, 1993). Estas especies son Catadysia rosulans Schulz, Draba ochropetala Schulz y Weberbauera smithii Al-Shehbaz. Adicionalmente se recolectaron otras dos especies restringidas al Perú, Lepidium kalenbornii Hitchc. y Weberbauera perforata Al-Shehbaz conocidas anteriormente para otros departamentos (Hitchcock, 1945; Al-Shehbaz, 1990). Tanto para $D$. ochropetala como $W$. perforata se confirma y amplia su distribución gracias a nuevas recolectas luego de su primer registro en 1920 y 1957 respectivamente.

De la poca frecuencia y dificultad para encontrar y recolectar las especies endémicas se puede deducir que estas se desarrollan en condiciones ambientales muy especializadas y con pocas posibilidades de dispersión que impiden que se incremente el número de individuos y poblaciones y por lo tanto su distribución. Tal es el caso de dos especies bastante 
raras como Aschersoniodoxa cachensis (Speg.) Al-Shehbaz y Englerocharis peruviana Musch. que se desarrollan a altitudes porencima de los 4500 $\mathrm{m}$ y sobre suelos crioturbados, formando poblaciones de no más de 15 ó 20 individuos. Para poder subsistir en tales condiciones, estas especies han desarrollado características como la suculencia en los órganos para el caso de $A$. cachensis, o la formación de tricomas alargados y rígidos de color plateado que reflejan la radiación solar excesiva como en el caso de E. peruviana y $W$. perforata.

Por el contrario, existen especies de amplia distribución geográfica y que se desarrollan en un amplio rango altitudinal como Cremolobus chilensis (Lag. ex DC) DC. y Capsella bursa-pastoris (L.) Hayek las cuales han desarrollado una enorme capacidad para adaptarse a diversos tipos de climas a través de la reducción en el tamaño de sus órganos y el incremento de la pubescencia.

Las 40 especies que se confirman para Ancash representan aproximadamente un tercio del total de especies de Brassicaceae que forman parte de la flora nacional. Debido al importante número de especies endémicas y a su restringida distribución es que es necesario continuar incrementando el conocimiento de esta familia a nivel nacional.

\section{Agradecimientos}

Los autores agradecen por el apoyo económico al Fondo de Desarrollo Universitario (FEDU) de la Universidad Nacional Mayor de San Marcos, a través del Instituto de Ciencias Biológicas «Antonio Raimondi» (ICBAR) de la Facultad de Ciencias Biológicas; y a la Compañía Minera Antamina, merced a un convenio con el Patronato del Museo de Historia Natural. Nuestra inmensa gratitud a los colegas Blanca León, José Roque, Irayda Salinas y María I. La Torre por la revisión y sugerencias al manuscrito.

\section{Literatura citada}

Albán J. 1998. Etnobotánica y conservación en la comunidad andina de Pamparomás, Huaylas, Ancash, Perú. Tesis para optar el grado académico de Magíster. UNMSM.

Al-Shehbaz I. 1990. Weberbauera perforata (Brassicaceae), ANew Species from Peru. Ann. Missouri Bot. Gard. 77: 841-842.

Brako L. \& J.L. Zarucchi. 1993. Catalogue of the Flowering Plants and Gymnosperms of Peru. Syst. Bot. Mongr. 45. Missouri Botanical Garden. 1286 pp.

Bridson D \& L. Forman. 1992. Herbarium Handbook. Royal Botanic Gardens, Kew.

Cano A. K.R. Young y B. León. 1996. Áreas importantes para la conservación de las fanerógamas en el Perú. pp. 39-46 en Rodríguez, L. Diversidad biológica del Perú: Zonas Prioritarias para su Conservación. Proyecto FANPE, GTZINRENA, Lima.

Cerrate E. 1969. Manera de preparar plantas para un herbario. Museo de Historia Natural. Serie de Divulgación N. ${ }^{\circ} 1$.

Cerrate E. 1979. Vegetación del Valle de Chiquián (departamento de Ancash). Edit. Los Pinos, Lima. 65 pp.

Cronquist A. 1981. An integrated system of classification of flowering plants. New York: Columbia University Press.

Hitchcock L. 1945. The South American Species of Lepidium. Lilloa 11: 74-134.

Lot E. y F. Chiang (Compiladores). 1986. Manual de Herbario. Consejo Nacional de la Flora de México. México. 42 pp.

Macbride J.F. 1938. Flora of Peru, part 1. Fieldiana: Bot. 13 (1): 1-170.

Monsalve C. y A. Cano 2003. La Familia Brassicaceae en la Provincia de Huaylas, Ancash. Rev. Peru. biol. 10(1): 20-32.

Peñaherrera C. 1969. Geografía General del Perú. Tomo I. Aspectos Físicos.

Sagástegui A. y S. Leiva. 1993. Flora invasora de los cultivos del Perú. Editorial Libertad, EIRL. Trujillo.

Smith D. 1988. Flora and Vegetation of the Huascaran National Park, Ancash, Peru; with preliminary studies for a manual of the flora. Thesis to obtain the degree of Doctor of Philosophy. Iowa State University, Ames, Iowa. 
Anexo 1. Lista de localidades de colecta

\begin{tabular}{|c|c|c|c|c|c|}
\hline \multicolumn{3}{|c|}{$\mathrm{N}^{\circ}$ PROVINCIA Localidad } & Coordenadas & \multicolumn{2}{|c|}{ Altitud Colectas } \\
\hline 1 & Aija & Aija & $9^{\circ} 43^{\prime} 18.9^{\prime \prime} \mathrm{S} \quad 77^{\circ} 29^{\prime} 19.2^{\prime \prime} \mathrm{O}$ & $3890-4150 m$ & $x$ \\
\hline 2 & Aija & Aija, abra de Huancapeti & $9^{\circ} 45^{\prime} 07.9^{\prime \prime} \mathrm{S} \quad 77^{\circ} 32^{\prime} 00^{\prime \prime} \mathrm{O}$ & $4490-4560 \mathrm{~m}$ & $x$ \\
\hline 3 & Aija & La Merced & $9^{\circ} 44^{\prime} 00.4^{\prime \prime S} \quad 77^{\circ} 36^{\prime} 59.5^{\prime \prime} \mathrm{O}$ & $3200-3500 \mathrm{~m}$ & $x$ \\
\hline 4 & $\begin{array}{l}\text { Antonio } \\
\text { Raimondi }\end{array}$ & Chingas & $9^{\circ} 07^{\prime} 09.9^{\prime \prime} \mathrm{S} \quad 76^{\circ} 57^{\prime} 43^{\prime \prime} \mathrm{O}$ & $2350-4300 \mathrm{~m}$ & $x$ \\
\hline 5 & $\begin{array}{l}\text { Antonio } \\
\text { Raimondi }\end{array}$ & Laguna Yanacocha & $77^{\circ} 04^{\prime} 31.1^{\prime \prime} \mathrm{O}$ & $4200-4300 \mathrm{~m}$ & $x$ \\
\hline 6 & Asunción & Chacas, abra de Punta Olímpica & $9^{\circ} 07^{\prime} 51.4^{\prime \prime S} \quad 77^{\circ} 30^{\prime} 37.2^{\prime \prime} \mathrm{O}$ & $\ldots$ & $x$ \\
\hline 7 & Asunción & Pompey & $9^{\circ} 09^{\prime} 43.7^{\prime \prime S} \quad 77^{\circ} 27^{\prime} 49.6^{\prime \prime O}$ & $3850 \mathrm{~m}$ & \\
\hline 8 & Bolognesi & Aquia & ..... & $4300-4430 \mathrm{~m}$ & $x$ \\
\hline 9 & Bolognesi & Capillpunta, al sur de Chiquián & $\ldots .$. & $3540 \mathrm{~m}$ & $x$ \\
\hline 10 & Bolognesi & Chiquián & $\ldots .$. & $3350 \mathrm{~m}$ & $x$ \\
\hline 11 & Bolognesi & Jerusalem, al este de Aquia & $\ldots .$. & $3200-3500 m$ & $x$ \\
\hline 12 & Bolognesi & Lihuan & $10^{\circ} 12^{\prime} 8.3^{\prime \prime} \mathrm{S} \quad 76^{\circ} 57^{\prime} 21.1^{\prime \prime} \mathrm{O}$ & $3870-3950 \mathrm{~m}$ & $x$ \\
\hline 13 & Bolognesi & Pocpa, Llamac, Pallca & $76^{\circ} 58^{\prime} 00^{\prime \prime} \mathrm{O}$ & $3350-3700 \mathrm{~m}$ & $x$ \\
\hline 14 & Bolognesi & Vista Alegre, valle de Chilca & $\ldots .$. & $3663 \mathrm{~m}$ & $x$ \\
\hline 15 & Casma & Lomas de Casma & $\ldots .$. & $400-500 \mathrm{~m}$ & $x$ \\
\hline 16 & Carhuáz & Carhuáz & $\ldots .$. & $3600-3650 m$ & $x$ \\
\hline 17 & Carhuáz & Parque Nacional Huascarán, Q. Ishi & $9^{\circ} 23^{\prime \prime \prime} \mathrm{S} \quad 77^{\circ} 28^{\prime \prime \prime} \mathrm{O}$ & $3900-4930 \mathrm{~m}$ & $x$ \\
\hline 18 & Carhuáz & Parque Nacional Huascarán, Ulta & $\ldots .$. & $3650 \mathrm{~m}$ & $x$ \\
\hline 19 & Carhuáz & Ponquez & $\ldots .$. & $2800-3600 \mathrm{~m}$ & $x$ \\
\hline 20 & $\begin{array}{l}\text { Carlos F. } \\
\text { Fitzcarrald }\end{array}$ & Yauya-San Francisco & $8^{\circ} 59^{\prime} 22.1^{\prime \prime} \mathrm{S} \quad 77^{\circ} 17^{\prime} 2522 \mathrm{O}$ & $3100-3300 \mathrm{~m}$ & $\mathrm{x}$ \\
\hline 21 & $\begin{array}{l}\text { Carlos F. } \\
\text { Fitzcarrald }\end{array}$ & Laguna Huachı & $9^{\circ} 10^{\prime} 06.4^{\prime \prime S} \quad 77^{\circ} 13^{\prime} 51.9^{\prime \prime} \mathrm{O}$ & 4200 & \\
\hline 22 & $\begin{array}{l}\text { Carlos F. } \\
\text { Fitzcarrald }\end{array}$ & San L & $9^{\circ} 07^{\prime} 13^{\prime \prime S} \quad 77^{\circ} 18^{\prime} 27^{\prime \prime} \mathrm{O}$ & $3500-3700 \mathrm{~m}$ & \\
\hline 23 & Corongo & Tarica & & & $x$ \\
\hline 24 & Huaraz & Olleros & $9^{\circ} 38^{\prime} 04.4^{\prime \prime S} \quad 77^{\circ} 29^{\prime} 35.8^{\prime \prime} \mathrm{O}$ & $3400 \mathrm{~m}$ & \\
\hline 25 & Huaraz & Parque Nac & $9^{\circ} 27^{\prime} \mathrm{S} \quad 77^{\circ} 27^{\prime} \mathrm{O}$ & $4400-4600 \mathrm{~m}$ & $x$ \\
\hline 26 & Huaraz & Parque Nacional Huascarán,Q. Shallap & $77^{\circ} 22^{\prime} \mathrm{O}$ & $3690-4100 \mathrm{~m}$ & $x$ \\
\hline 27 & Huaraz & Punta Callán & $\ldots .$. & $4200-4400 \mathrm{~m}$ & $x$ \\
\hline 28 & Huari & Huari, alrededores de la Laguna Puruhuay & $9^{\circ} 19^{\prime} 11.2^{\prime \prime} \mathrm{S}$ & $3400-3500 \mathrm{~m}$ & \\
\hline 29 & Huari & Huari, Tashta & $77^{\circ} 12^{\prime} 28.7^{\prime \prime} \mathrm{O}$ & $3900-4100 \mathrm{~m}$ & \\
\hline 30 & Huari & Juprog, Campamento minero Antamina & $\ldots .$. & $3800 \mathrm{~m}$ & $x$ \\
\hline 31 & Huari & Parque Nacional Huascarán, Tunel Cahuish & $77^{\circ} 14^{\prime} \mathrm{O}$ & $4350-4550 \mathrm{~m}$ & $x$ \\
\hline 32 & Huari & Ponto & $77^{\circ} 00^{\prime} 24.3^{\prime \prime} \mathrm{O}$ & $3350 \mathrm{~m}$ & $x$ \\
\hline 33 & Huari & San Marcos & $\ldots .$. & $4600-4800 \mathrm{~m}$ & $x$ \\
\hline 34 & Huari & Ucco, $\mathrm{m}$ & $9^{\circ} 11^{\prime} 34.2^{\prime \prime} \mathrm{S} \quad 76^{\circ} 54^{\prime} 22.2^{\prime \prime} \mathrm{O}$ & $3900-4100 \mathrm{~m}$ & \\
\hline 35 & Huari & Tinco & $76^{\circ} 58^{\prime} 48.3^{\prime \prime} \mathrm{O}$ & $3850-3900 \mathrm{~m}$ & \\
\hline 36 & Huari & Yanace & $9^{\circ} 34^{\prime} 34.7^{\prime \prime S} \quad 77^{\circ} 02^{\prime} 57.6^{\prime \prime O}$ & $4150-4820 \mathrm{~m}$ & $x$ \\
\hline 37 & Huaylas & Abra Tres Cruces, & $\ldots .$. & $4400-4600 \mathrm{~m}$ & $x$ \\
\hline 38 & Huaylas & Caráz, San Pedro & $\ldots .$. & $2200 \mathrm{~m}$ & $x$ \\
\hline 39 & Huaylas & Cerro Rico, Pampa & $9^{\circ} 03^{\prime} 58.4^{\prime \prime S} \quad 77^{\circ} 55^{\prime} 20.8^{\prime \prime} \mathrm{O}$ & $4400-4800 \mathrm{~m}$ & $x$ \\
\hline 40 & Huaylas & Cerro Yanaico, Pueblo Libre & $9^{\circ} 06^{\prime} 47^{\prime \prime} \mathrm{S} \quad 77^{\circ} 48^{\prime} 32^{\prime \prime} \mathrm{O}$ & $2380-2650 \mathrm{~m}$ & $x$ \\
\hline 41 & Huaylas & Huashta Cruz, Pueblo Libre & .... & $4000-4200 \mathrm{~m}$ & $x$ \\
\hline 42 & Huaylas & La Hoyada, Pueblo Libre & $9^{\circ} 09^{\prime} 58^{\prime \prime S} 77^{\circ} 47^{\prime} 30^{\prime \prime} \mathrm{O}$ & $2600-3050 \mathrm{~m}$ & $x$ \\
\hline 43 & Huaylas & Laguna Parón, Caraz & ..... & $4100-4400 \mathrm{~m}$ & $x$ \\
\hline 44 & Huaylas & Ocshapampa, Pampar & $9^{\circ} 11^{\prime} 29.7^{\prime \prime S} \quad 77^{\circ} 51^{\prime} 50.7^{\prime \prime O}$ & $4350-4630 m$ & $x$ \\
\hline 45 & Huaylas & Parque Nacional Huas & $8^{\circ} 53^{\prime} \mathrm{S} \quad 77^{\circ} 41^{\prime} \mathrm{O}$ & $4600-4750 \mathrm{~m}$ & $x$ \\
\hline 46 & Huaylas & Parque Nacional Huascarán, Q. Los Cedros & $8^{\circ} 51^{\prime} \mathrm{S} \quad 77^{\circ} 45^{\prime} \mathrm{O}$ & $4600-4850 \mathrm{~m}$ & $x$ \\
\hline 47 & Huaylas & Parque Nacional Huascarán, Q. Santa Cruz & $8^{\circ} 55^{\prime} \mathrm{S} \quad 77^{\circ} 36^{\prime} \mathrm{O}$ & $2700-4800 \mathrm{~m}$ & $x$ \\
\hline 48 & Huaylas & Parque Nacional Huascarán, R. Auquispuquio & $8^{\circ} 50^{\prime} \mathrm{S} \quad 77^{\circ} 38^{\prime} \mathrm{O}$ & $3750-3900 \mathrm{~m}$ & $x$ \\
\hline 49 & Huaylas & Pueblo Libre & $9^{\circ} 06^{\prime} 47^{\prime \prime S} \quad 77^{\circ} 48^{\prime} 32^{\prime \prime} \mathrm{O}$ & $2380-2450 \mathrm{~m}$ & $x$ \\
\hline 50 & Huaylas & Q. Cachicoral, Pamparomás & $9^{\circ} 09^{\prime} 10^{\prime \prime} \mathrm{S} \quad 77^{\circ} 52^{\prime} 06^{\prime \prime} \mathrm{O}$ & $3900-4400 \mathrm{~m}$ & $x$ \\
\hline 51 & Huaylas & Riurín & $\ldots .$. & $4200-4620 \mathrm{~m}$ & $x$ \\
\hline 52 & Huaylas & Santo Toribio & & $2900 \mathrm{~m}$ & $x$ \\
\hline 53 & $\begin{array}{l}\text { Mariscal } \\
\text { Luzuriag }\end{array}$ & Carretera Llumpa-Piscobamba & $77^{\circ} 22^{\prime} 06.3^{\prime \prime} \mathrm{O}$ & $2700-3100 \mathrm{~m}$ & \\
\hline 54 & Pomabamb & a Por & & $3700-4000 \mathrm{~m}$ & \\
\hline 55 & Recuay & Marca & $\ldots .$. & $2600 \mathrm{~m}$ & $x$ \\
\hline
\end{tabular}




\begin{tabular}{|c|c|c|c|c|c|}
\hline 56 Recuay & $\begin{array}{l}\text { Parque Nacional Huascarán, } \\
\text { entre Pastoruri y Raria }\end{array}$ & $9^{\circ} 52^{\prime} \mathrm{S}$ & $77^{\circ} 11^{\prime} \mathrm{O}$ & $4700-4800 \mathrm{~m}$ & $x$ \\
\hline 57 Recuay & Parque Nacional Huascarán, Q. Quenua Ragra & $9^{\circ} 58^{\prime} \mathrm{S}$ & $77^{\circ} 13^{\prime} \mathrm{O}$ & $4600-4850 \mathrm{~m}$ & $x$ \\
\hline 58 Recuay & Parque Nacional Huascarán, Q. Queshque & $9^{\circ} 50^{\prime} \mathrm{S}$ & $77^{\circ} 18^{\prime} \mathrm{O}$ & $4500-4600 \mathrm{~m}$ & $x$ \\
\hline 59 Recuay & Parque Nacional Huascarán, Río Pachacoto & $9^{\circ} 52^{\prime} \mathrm{S}$ & $77^{\circ} 17^{\prime} \mathrm{O}$ & $4200-4720 \mathrm{~m}$ & $x$ \\
\hline 60 Recuay & Parque Nacional Huascarán, Runa Shimi & $9^{\circ} 53^{\prime} \mathrm{S}$ & $77^{\circ} 17^{\prime} \mathrm{O}$ & $4200-4300 \mathrm{~m}$ & $\mathrm{x}$ \\
\hline 61 Santa & Macate & $\ldots$. & & $3100-3310 m$ & $\mathrm{x}$ \\
\hline 62 Yungay & Parque Nacional Huascarán, Sector Llanganuco & $9^{\circ} 01^{\prime} \mathrm{S}$ & $77^{\circ} 36^{\prime} \mathrm{O}$ & $3580-5080 \mathrm{~m}$ & $x$ \\
\hline 63 Yungay & Parque Nacional Huascarán, Q. Ranincuray & $8^{\circ} 59^{\prime} \mathrm{S}$ & $77^{\circ} 34^{\prime} \mathrm{O}$ & $4100-4200 \mathrm{~m}$ & $x$ \\
\hline 4 Yungay & Yauya & $9^{\circ} 02^{\prime} \mathrm{S}$ & $77^{\circ} 36^{\prime} \mathrm{O}$ & $3500-3800 m$ & $x$ \\
\hline
\end{tabular}

$\mathrm{Q}=$ quebrada, $\mathrm{R}=$ ruinas

Anexo 2. Listado de especies de Brassicaceae presentes en Ancash. Especies endémicas (E)

\begin{tabular}{|c|c|c|}
\hline Tribu & Género & Nombre Científico \\
\hline Arabidae & $\begin{array}{l}\text { Aschersoniodoxa } \\
\text { Brayopsis } \\
\text { Cardamine } \\
\text { Catadysia } \\
\text { Draba }\end{array}$ & $\begin{array}{l}\text { Aschersoniodoxa cachensis (Speg.) Al-Shehbaz } \\
\text { Brayopsis alpaminae subsp. smithii Al-Shehbaz } \\
\text { Brayopsis calycina (Desv.) Gilg \& Muschl. } \\
\text { Cardamine bonariensis DC. } \\
\text { Catadysia rosulans O. E. Schulz }{ }^{(\mathrm{E})} \\
\text { Draba alchemilloides Gilg } \\
\text { Draba alyssoides Humb. \& Bonp. ex DC. } \\
\text { Draba brackenridgei A. Gray } \\
\text { Draba cryptantha Hook. } \\
\text { Draba depressa Hook. } \\
\text { Draba hemsleyana Gilg } \\
\text { Draba macleanii Hook. } \\
\text { Draba ochropetala O. E. Schulz }{ }^{(\mathrm{E})} \\
\text { Draba pickeringii A. Gray } \\
\text { Englerocharis peruviana Musch. } \\
\text { Nasturtium officinale } \text { R. Br. }\end{array}$ \\
\hline Brassiceae & $\begin{array}{l}\text { Brassica } \\
\text { Raphanus }\end{array}$ & $\begin{array}{l}\text { Brassica rapa subsp. campestris (L.) Clapham } \\
\text { Brassica juncea (L.) Coss. } \\
\text { Brassica nigra (L.) Koch. } \\
\text { Raphanus raphanistrum L. } \\
\text { Raphanus sativus L. }\end{array}$ \\
\hline Cremolobeae & Cremolobus & Cremolobus chilensis (Lag. ex DC.) DC. \\
\hline Lepideae & $\begin{array}{l}\text { Capsella } \\
\text { Lepidium }\end{array}$ & $\begin{array}{l}\text { Capsella bursa-pastoris (L.) Medik. } \\
\text { Lepidium bipinnatifidum Desv. } \\
\text { Lepidium chichicara Desv. } \\
\text { Lepidium kalenbornii Hitchc. }{ }^{(\mathrm{E})} \\
\text { Lepidium meyenii Walp. } \\
\text { Lepidium pubescens Desv. } \\
\text { Lepidium virginicum L. }\end{array}$ \\
\hline Sisymbrieae & $\begin{array}{l}\text { Halimolobus } \\
\text { Mancoa } \\
\text { Sisymbrium } \\
\text { Weberbauera }\end{array}$ & 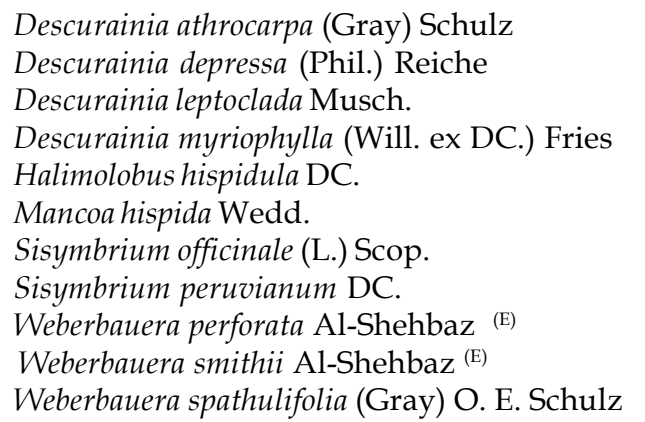 \\
\hline
\end{tabular}

(E) Especies endémicas 